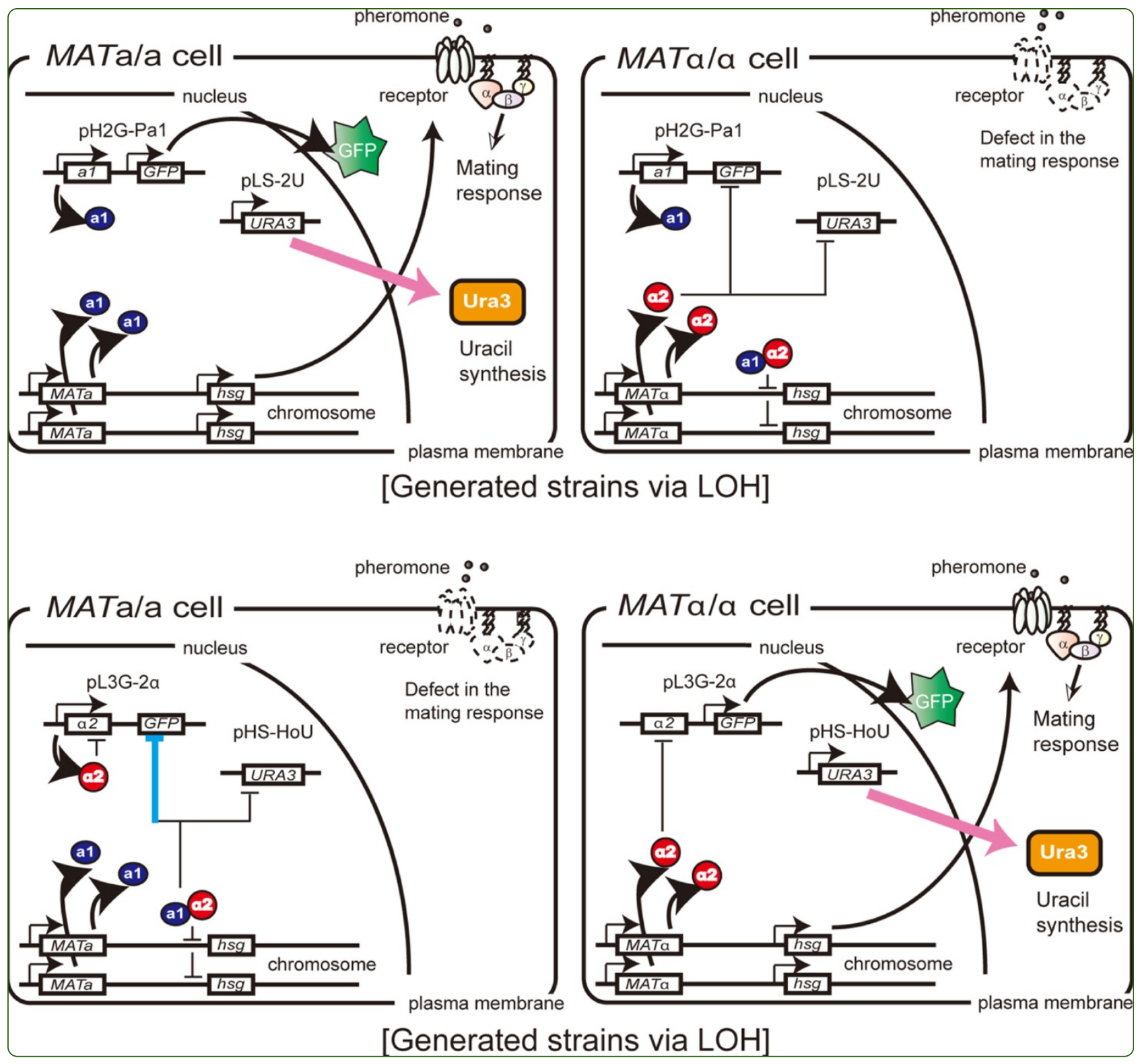

\title{
Development of growth selection systems to isolate a-type or a-type of yeast cells spontaneously emerging from MATa/a diploids
}

Fukuda and Honda 


\title{
Development of growth selection systems to isolate a-type or a-type of yeast cells spontaneously emerging from MATa/a diploids
}

Nobuo Fukuda and Shinya Honda*

\begin{abstract}
Background: Manufacture of MATa and MATa yeast cells is required for crossbreeding, a procedure that permits hybridization and the generation of new heterozygous strains. Crossbreeding also can be performed with a- and a-type of cells, which have the same mating abilities as MATa and MATa haploid cells, respectively.

Results: In this work, we describe a method to generate a- and a-type of cells via the naturally-occurring chromosomal aberration in parental MATa/a diploids. We successfully designed suitable genetic circuits for expression of the URA3 selection marker gene to permit isolation of a- and a-type of cells, respectively, on solid medium lacking uracil. Furthermore we succeeded in generation of zygotes by mating of both the manufactured a- and a-type of yeast cells.

Conclusions: This process does not require exposure to mutagens such as UV irradiation, thereby avoiding the accumulation of undesirable mutations that would detract from the valuable traits that are under study. All the genetic modifications in the current study were introduced into yeast cells using plasmids, meaning that these traits can be removed without altering the genome sequence. This approach provides a reliable and versatile tool for scientific research and industrial yeast crossbreeding.
\end{abstract}

Keywords: Yeast, Biotechnology, Gene regulation, Transcription factors, Promoters

\section{Background}

Crossbreeding is an effective approach used to improve and combine traits of yeast strains by zygosis of cells of

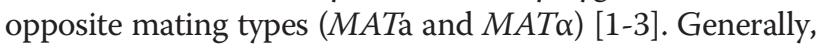
parental diploid and polyploid strains used for industrial application are never able to mate directly, and hence isolation of mating strains (typically, haploid strains) via sporulation is a prerequisite for crossbreeding. Yet classical crossbreeding can be problematic because numerous industrial yeast strains sporulate poorly or not at all [4-8].

The MAT genes locate on chromosome III that is the most unstable one among 16 chromosomes of Saccharomyces cerevisiae [9]. Naturally, a- and $\alpha$-type of yeast cells emerge from $M A T \mathrm{a} / \alpha$ diploids with quite low frequency, due to chromosomal aberration during mitotic division. These cells can be used as alternative mating

\footnotetext{
*Correspondence: s.honda@aist.go.jp

Biomedical Research Institute, National Institute of Advanced Industrial Science and Technology (AIST), Higashi, Tsukuba, Ibaraki 305-8566, Japan
}

strains, possessing the same mating ability as MATa and $M A T \alpha$ haploids generated via sporulation, respectively.

There are several kinds of chromosomal aberration such as loss of heterozygosity ( $\mathrm{LOH}$ ) and mitotic chromosome loss. $\mathrm{LOH}$ is a natural event that generates homozygous loci via chromosomal rearrangement in heterozygous loci [10-13], and LOH occurring at the MAT locus within a $M A T a / \alpha$ cell produces either a MATa $/ \mathrm{a}$ or a $M A T \alpha / \alpha$ cell. The spontaneous frequency of LOH is below $1 \times 10^{-4}$ [14]. Mitotic chromosome loss is also a naturally-occurring event that polyploid cells lose single or multiple chromosomes [15]. The frequency of loss of chromosome III in yeast diploid cells was reported to be $5 \times 10^{-5}$ [9], and yeast cells having lost one of two chromosomes III containing the MATa or MAT $\alpha$ gene acquire either a- or $\alpha$-type of mating ability. Unfortunately, however, it can be difficult to isolate the generated a- and $\alpha$-type of cells, since the spontaneous frequencies of such events are quite low.

Ultraviolet (UV) irradiation of yeast diploid cells has been used successfully to increase the frequency of $\mathrm{LOH}$ 
to about 30\% [16]. According to other report, chemicals such as benzonitrile and methyl ethyl ketone increase the frequency of chromosome loss [17]; however, these treatments have the potential to randomly induce undesirable mutations at loci other than the MAT locus or undesirable loss of chromosomes other than chromosome III, which might prevent the generated a- or $\alpha$ type of cells from inheriting the desired properties of the parental strains when used in crossbreeding.

Here we designed genetic circuits to express the URA3 gene as a selection marker, permitting isolation of the rare a- or $\alpha$-type of cells arising via spontaneous chromosomal aberration (Figure 1). Furthermore, we introduced a previously constructed expression system to prevent autopolyploidization between a- and $\alpha$-type of cells generated from the same parental strain. Specifically, we used the a1- $\alpha 2$ complex [18], a repressor of haploid-specific genes $(h s g)$ that endow yeast cells with the ability to mate [19]. In this work, we show the feasibility of this approach and its potential to provide mating strains for yeast crossbreeding.

\section{Results}

\section{General strategy}

An outline of the experimental design for production of a- and $\alpha$-type of cells is shown in Figure 1. Spontaneous chromosomal aberration during cultivation provide low numbers of both a- and $\alpha$-type of cells in cultures containing large numbers of parental MATa/ $\alpha$ cells. The inclusion in our system of the a1- $\alpha 2$ complex (which represses expression of $h s g$ ) suppresses mating by the rare a- and $\alpha$-type of cells, which therefore can grow independently in the cell mixture without forming nonhybrid (autopolyploid) cells [18]. Here we used URA3 as the selection marker gene for a- (Figure 1A) or $\alpha$-type of cells (Figure 1B), permitting isolation by selection on solid medium lacking uracil (SD - Ura plates).

All the genetic modifications in the current study were introduced into yeast cells using plasmids, permitting complete removal of the modification following isolation of the target cells. Final plasmid sets suitable for isolation of a- or $\alpha$-type of cells were defined by sequential

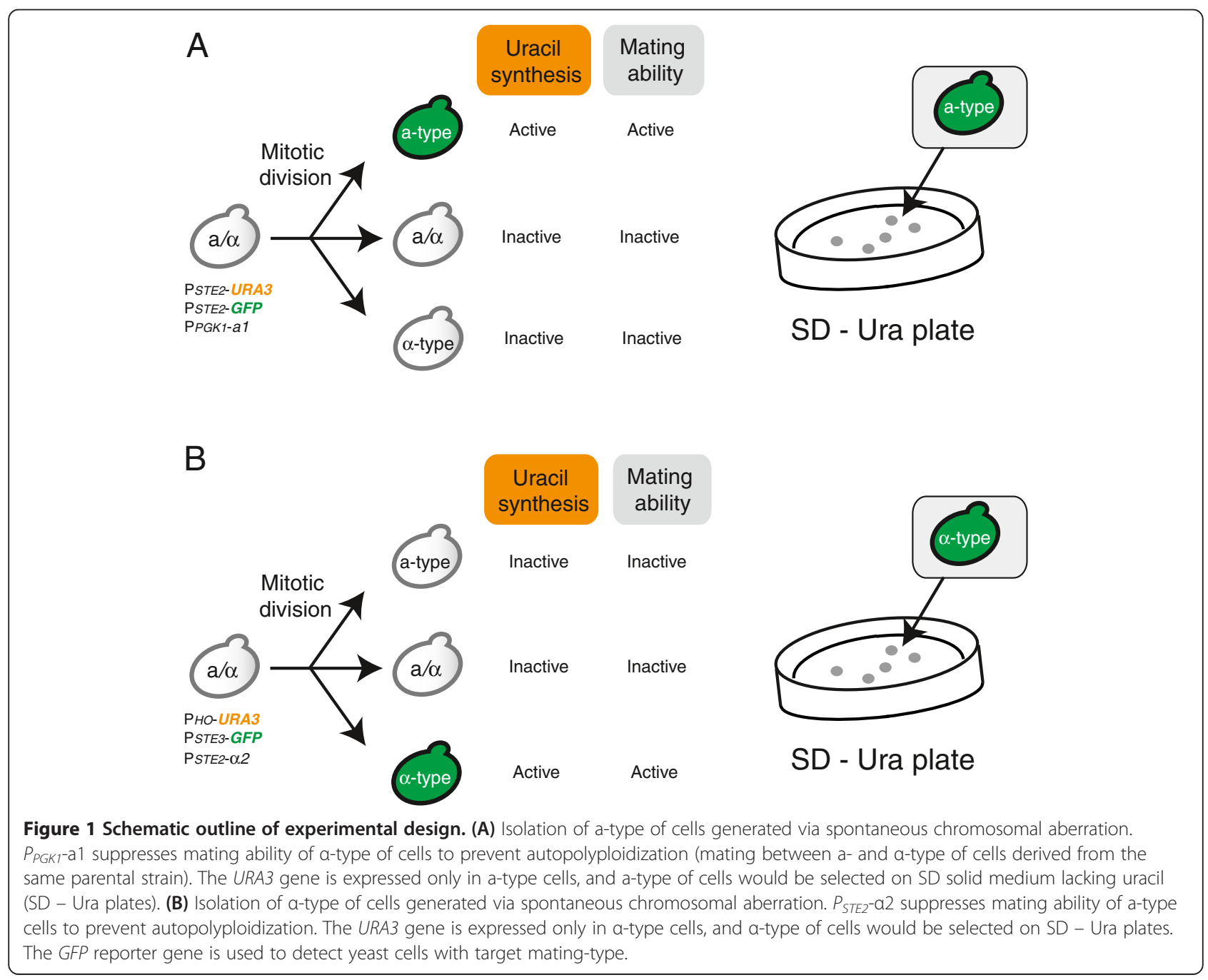


trial and error. The process is described below in a stepby-step manner.

Trial 1: expression of the URA3 gene via YEp-type plasmids Two kinds of YEp-type (multi-copy) plasmids were constructed to express the URA3 gene in a- or $\alpha$-type of cells (Figure 2). Plasmid pHY-2U (Figure 2A) places the selection marker under control of $P_{S T E 2}$, a promoter of a-typespecific genes (asg), so that only MATa cells can express the URA3 gene. On the other hand, plasmid pLY-3U (Figure 2C) places the selection marker under control of $P_{S T E 3}$, a promoter of $\alpha$-type-specific genes $(\alpha s g)$, so that

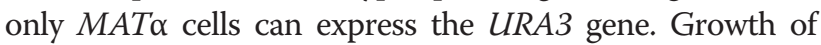
each transformant was evaluated by monitoring the $\mathrm{OD}_{600}$.

Only MATa cells possessing pHY-2U grew without uracil at the same level as with uracil (Figure 2B). Although there were obvious differences in growth ability between MATa cells and others, MAT $\alpha$ and MATa/ $\alpha$ cells harboring pHY-2U exhibited some growth in the absence of uracil. As shown in Figure 2D, MATa and $M A T a / \alpha$ cells harboring pLY-3U exhibited levels of growth equivalent to that of MAT $\alpha$ cells harboring pLY$3 \mathrm{U}$ when cultured on SD - Ura. These results suggest that leaky expression of the URA3 gene via YEp-type of plasmids would preclude isolation of target cells on SD Ura plates.
Trial 2: expression of the URA3 gene via YCp-type plasmids To reduce leaky URA3 gene expression, DNA fragments containing $P_{S T E 2}-U R A 3$ or $P_{S T E 3}-U R A 3$ were transferred into YCp-type (single-copy) plasmids to yield pLS-2U (Figure 3A) or pHS-3U (Figure 3C), respectively. To make detailed examination of changes in growth ability of yeast cells, we tracked $\mathrm{OD}_{600}$ values over time, and then plotted growth curves for each transformant with or without uracil. Whereas pLS-2U permitted MATa cells to grow without uracil, $M A T \alpha$ and $M A T a / \alpha$ cells harboring pLS-2U exhibited only minimal growth in the absence of uracil (Figure 3B). These results suggested that pLS-2U is suitable for isolation of a-type of cells.

Among cells transformed with pHS-3U, MATa and $M A T$ a/ $\alpha$ grew poorly without uracil compared to $M A T \alpha$ (Figure 3D). Although the leakiness of URA3 gene expression was significantly reduced by altering plasmid type from YEp to YCp, further optimization was needed to establish an isolation method for $\alpha$-type of cells.

Trial 3: use of the a1-a2 repressor complex combined with $P_{H O}$, a promoter of $h s g$, for a-type-specific URA3 gene expression

Two plasmids shown in Figure 4A were used in an improved strategy for $\alpha$-type-specific URA3 gene expression. The plasmid pL3G-2 $\alpha$ that contains $P_{S T E 2}-\alpha 2$
A
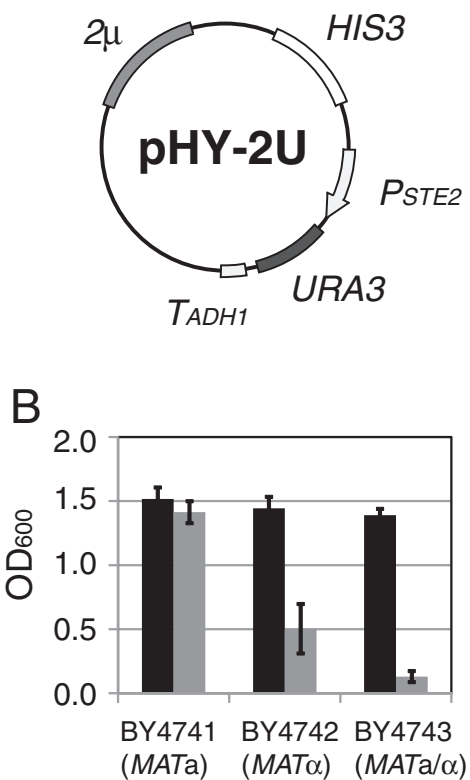

C
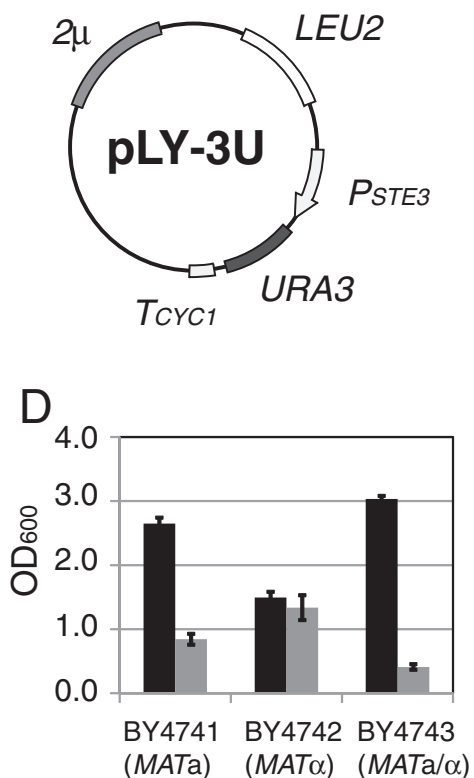

Figure 2 Growth of yeast transformants harboring YEp-type plasmids. (A) Plasmid map of pHY-2U containing $2 \mu$ origin of replication

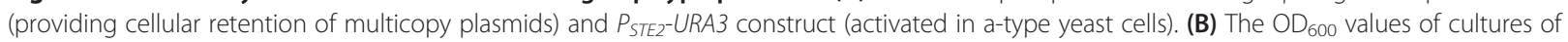
$\mathrm{pHY}-2 \mathrm{U}$ transformants at $18 \mathrm{~h}$ cultivation. Black bars indicate cultivation with uracil, and gray bars indicate cultivation without uracil. Values are presented as means \pm standard deviations from three independent experiments. (C) Plasmid map of pLY-3U containing $2 \mu$ origin of replication and $P_{\text {STE3 }}$ URA3 construct (activated in a-type yeast cells). (D) The OD 600 values of cultures of pLY-3U transformants at 18 h cultivation. Symbols are as in $B$. 

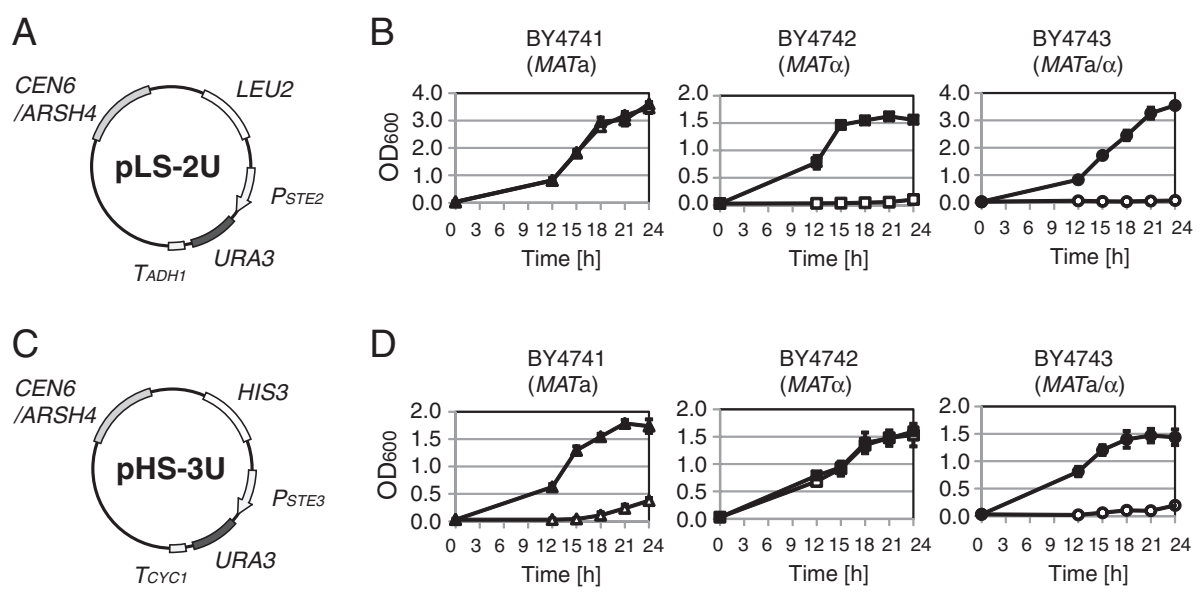

Figure 3 Growth of yeast transformants harboring YCp-type of plasmids. (A) Plasmid map of pLS-2U containing CEN6/ARSH4 origin of replication (providing cellular retention of single-copy plasmids) and $P_{\text {STE2 }}-U R A 3$ construct (activated in a-type yeast cells). (B) The growth curves of pLS-2U transformants. Closed symbols indicate cultivation with uracil, and open symbols indicate cultivation without uracil. Values are presented as means \pm standard deviations from three independent experiments. (C) Plasmid map of pHS-3U containing CEN6/ARSH4 origin of replication and $P_{\text {STE3 }}-U R A 3$ contruct (activated in a-type yeast cells). (D) The growth curves of pHS-3U transformants. Symbols are as in B.

suppresses the mating ability of MATa cells by artificially forming the a1- $\alpha 2$ repressor complex [18]. Because the a1- $\alpha 2$ complex can directly repress gene expression under the control of promoters of $h s g$, we selected $P_{H O}$ (a $h s g$ promoter) as an alternative promoter to express the $U R A 3$ gene. Hence, as shown in Figure $1 \mathrm{~B}, P_{H O^{-}}$ URA3 is expected to be activated only in MATa cells, where $P_{S T E 2^{-}} \alpha 2$ can drive expression off the $H O$ promoter.

Figure 4B shows growth curves of transformants in the improved strategy. As expected, while MAT $\alpha$ transformants grew without uracil, MATa and MATa/ $\alpha$ transformants exhibited minimal or no growth without uracil. These results suggest that use of pHS-HoU

\section{A}
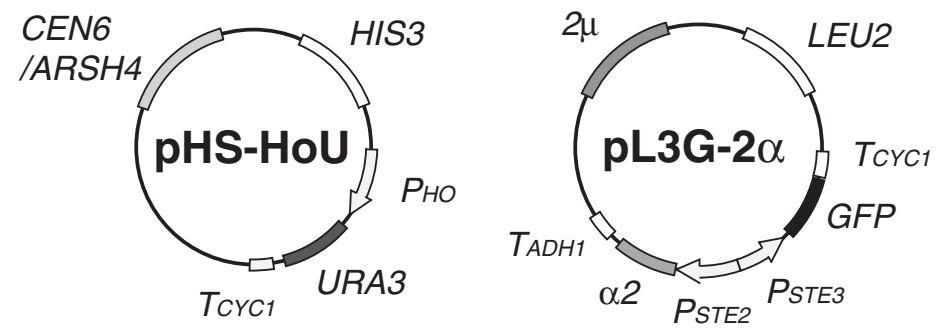

B

BY4741

(MATa)

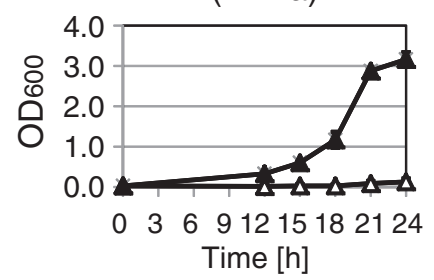

BY4742

$(M A T \alpha)$

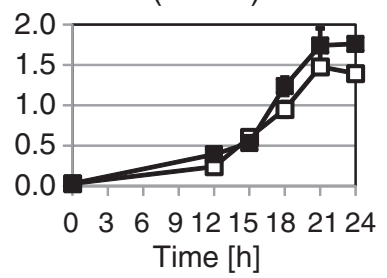

BY4743

$(M A T a / \alpha)$

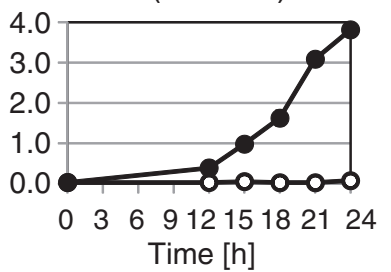

Figure 4 Improved growth selection system for a-type of cells. (A) Plasmids used for a-type-specific URA3 gene expression. The plasmid pHS-HoU contains CEN6/ARSH4 origin of replication (providing cellular retention of single-copy plasmids) and $P_{H_{O}}$-URA3 construct (activated in yeast haploid cells). The plasmid pL3G-2a contains $P_{\text {STE2 }}-\mathrm{a} 2$, a construct that represses URA3 gene expression in a-type yeast cells. (B) The growth curves of double plasmid (pHS-HoU and pL3G-2a) transformants. Closed symbols indicate cultivation with uracil, and open symbols indicate cultivation without uracil. Values are presented as means \pm standard deviations from three independent experiments. 
combined with pL3G-2 $\alpha$ is suitable for isolation of $\alpha$ type of cells.

\section{Artificial regulation network designed for isolation of a- and a-type of cells}

As described above, the plasmid pLS-2U (constructed in trial 2) was preferred for isolation of a-type of cells, and the combination of plasmids pHS-HoU and pL3G-2 $\alpha$ (as described in trial 3) was preferred for isolation of $\alpha$-type of cells. A model that describes how our artificial gene network can function in isolation of a- or $\alpha$-type of cells is shown in Figure 5.

For isolation of a-type of cells, we introduced plasmid pLS-2U into the parental strain in combination with plasmid pH2G-Pa1 (see Additional file 1: Figure S1A and $\mathrm{B}$ ), which prevents autopolyploidization (Table 1 and Figure 5A). The a1- $\alpha 2$ complex represses expression of $h s g$ that are required for mating signal transduction in $\alpha$-type of cells generated via chromosomal aberration, as well as in parental MATa $/ \alpha$ cells. In this system, only atype of cells can survive on SD - Ura plates, because of atype-specific URA3 gene expression. To confirm the mating-type of yeast cells isolated on SD - Ura plates using this method, we used a GFP reporter gene under the control of the a-type-specific promoter [15]. GFPfluorescence was observed in these yeast isolates, verifying the a-type, and confirming that this system can distinguish positives from false-positives in the scheme proposed in Figure 1A.

Next, a plasmid set composed of pHS-HoU and pL3G$2 \alpha$ was introduced into the parental strain for isolation of $\alpha$-type of cells. pL3G-2 $\alpha$ also provides the autopolyploidization prevention function (Table 1 and Figure 5B). The resulting a1- $\alpha 2$ complex represses expression of $h s g$ in a-type of cells as well as in the parental MATa/ $\alpha$ cells. In this system, only $\alpha$-type of cells can survive on SD Ura plates, because of $\alpha$-type-specific URA3 gene expression. To confirm the mating-type of isolated yeast
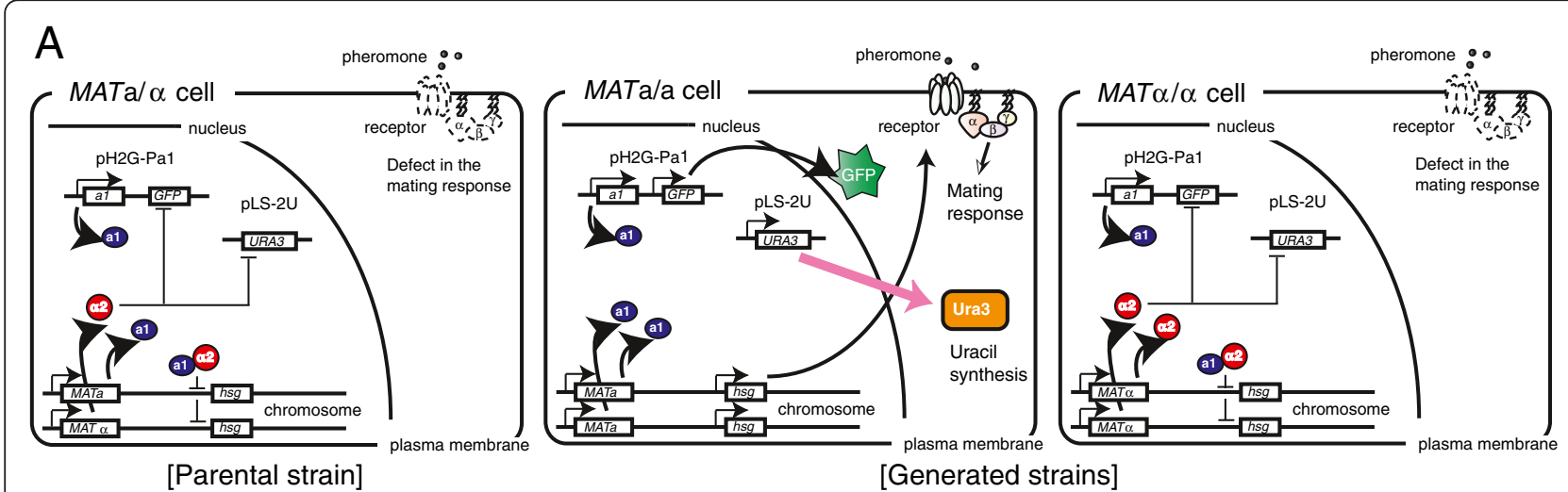

[Generated strains]

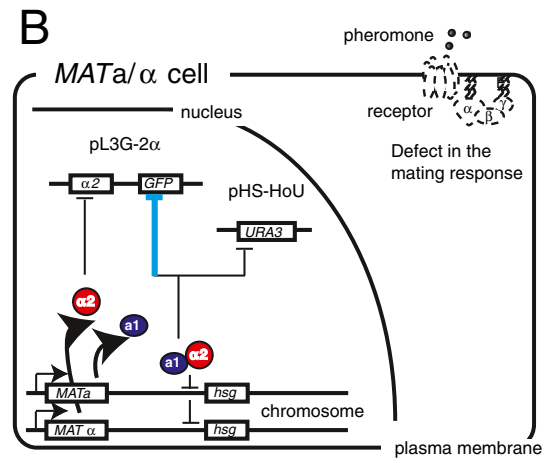

[Parental strain]
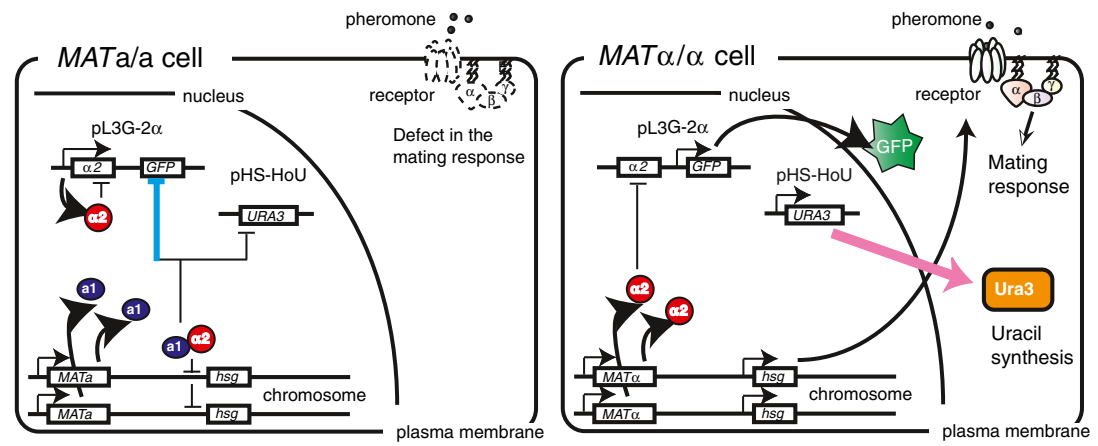

[Generated strains]

Figure 5 Schematic outline of yeast mating-type regulation and our engineered approach. (A) Engineering for isolation of a-type of cells. The MATa/a parental strain indicates BY4743 harboring pH2G-Pa1 and pLS-2U (see Table 1). (B) Engineering for isolation of a-type of cells. The MATa/a parental strain indicates BY4743 harboring pL3G-a2 and pHS-HoU (see Table 1). Chromosomal aberration regarding the MAT locus induces generation of two kinds of yeast cells, i.e., a- and a-type of cells. Here we described yeast cells possessing two sets of MAT genes (generated via $\mathrm{LOH}$ ) as an example. Then, spontaneous or engineered formation of the a1-a2 complex suppresses the mating response by repressing expression of haploid-specific genes ( $h s g$ ), which encode components of the mating signaling pathway. Target cells expressing the URA3 selection marker gene can be isolated on SD - Ura plates in the absence of autopolyploidization. The target cells also express the GFP reporter gene. Regulation colored with cyan indicates indirect gene repression; the a1-a2 complex prevents expression of transcriptional activator a1 that is required for gene expression under the control of $P_{\text {STE3 }}$. Note that URA3 gene is directly repressed by a transcriptional repressor in non-target cells. 
Table 1 Yeast strains and plasmids used in this study

\begin{tabular}{|c|c|c|}
\hline Name & Description & $\begin{array}{l}\text { Reference } \\
\text { source }\end{array}$ \\
\hline \multicolumn{3}{|l|}{$\begin{array}{l}\text { Yeast } \\
\text { strains }\end{array}$} \\
\hline BY4741 & MATa his $3 \Delta 1$ ura $3 \Delta 0$ leu $2 \Delta 0$ met $15 \Delta 0$ & $\begin{array}{l}\text { Brachmann } \\
\text { et al., [24] }\end{array}$ \\
\hline BY4742 & MATa his $3 \Delta 1$ ura3 $\Delta 0$ leu2 20 lys $2 \Delta 0$ & $\begin{array}{l}\text { Brachmann } \\
\text { et al., }[24]\end{array}$ \\
\hline BY4743 & 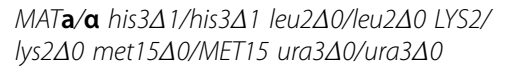 & $\begin{array}{l}\text { Brachmann } \\
\text { et al., [24] }\end{array}$ \\
\hline BY4743A & 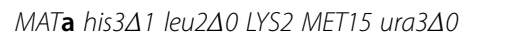 & Present study \\
\hline BY4743AL & $\begin{array}{l}\text { MATa/a his } 3 \Delta 1 / \text { his } 3 \Delta 1 \text { leu2 } \Delta 0 / \text { leu2 } \Delta 0 \text { LYS2/ } \\
\text { lys } 2 \Delta 0 \text { met15 } \Delta 0 / M E T 15 \text { ura3 } \Delta 0 / \text { ura3 } \Delta 0\end{array}$ & Present study \\
\hline \multicolumn{3}{|l|}{ Plasmids } \\
\hline pRS313 & $\begin{array}{l}\text { Yeast expression vector; CEN6/ARSH4 ori and } \\
\text { HIS3 marker }\end{array}$ & $\mathrm{NBRP}^{*}$ \\
\hline pRS315 & $\begin{array}{l}\text { Yeast expression vector; CEN6/ARSH4 ori and } \\
\text { LEU2 marker }\end{array}$ & NBRP \\
\hline pRS316 & $\begin{array}{l}\text { Yeast expression vector; CEN6/ARSH4 ori and } \\
\text { URA3 marker }\end{array}$ & NBRP \\
\hline $\mathrm{pHY}-2 \mathrm{GA}$ & $\begin{array}{l}\text { Yeast expression vector; } 2 \mu \text { ori, HIS3 marker } \\
\text { and } P_{\text {STE2 }} \text { EGFP }\end{array}$ & $\begin{array}{l}\text { Fukuda et al., } \\
{[18]}\end{array}$ \\
\hline pLY-3GC & $\begin{array}{l}\text { Yeast expression vector; } 2 \mu \text { ori, } L E U 2 \text { marker } \\
\text { and } P_{\text {STE3 }} \text { EGFP }\end{array}$ & $\begin{array}{l}\text { Fukuda et al., } \\
\text { [18] }\end{array}$ \\
\hline $\begin{array}{l}\mathrm{pH} 2 \mathrm{G}- \\
\mathrm{Pa} 1\end{array}$ & $P_{P G K 1}-\mathrm{a} 1$ in $\mathrm{pHY}-2 \mathrm{GA}$ & $\begin{array}{l}\text { Fukuda et al., } \\
\text { [18] }\end{array}$ \\
\hline pL3G-2a & $P_{\text {STE2- }}$ - 2 in $\mathrm{pLY}-3 \mathrm{GC}$ & $\begin{array}{l}\text { Fukuda et al., } \\
{[18]}\end{array}$ \\
\hline $\mathrm{pHY}-2 \mathrm{U}$ & $\begin{array}{l}\text { Yeast expression vector; } 2 \mu \text { ori, HIS3 marker } \\
\text { and } P_{\text {STE2 }} \text { URA3 }\end{array}$ & Present study \\
\hline$p L Y-3 U$ & $\begin{array}{l}\text { Yeast expression vector; } 2 \mu \text { ori, LEU2 marker } \\
\text { and } P_{\text {STE3 }} \text { URA3 }\end{array}$ & Present study \\
\hline pLS-2U & $P_{\text {STE2 }}-U R A 3$ in pYO315 & Present study \\
\hline pHS-3U & $P_{\text {STE3 }}-U R A 3$ in pYO313 & Present study \\
\hline pHS-HoU & $P_{H O}-U R A 3$ in $\mathrm{pYO} 313$ & Present study \\
\hline
\end{tabular}

"Resources were provided by the National Bio-Resource Project (NBRP) of the MEXT, Japan.

cells, we used a GFP reporter gene under the control of the $\alpha$-type-specific promoter [18]. GFP-fluorescence was observed in these yeast isolates, verifying the $\alpha$-type, and confirming the validity of the scheme proposed in Figure 1B.

\section{Isolation of a- and a-type of cells generated via spontaneous chromosomal aberration}

As shown in Figure 1A, MATa/ $\alpha$ cells possessing both plasmids pLS-2U and pH2G-Pa1 were washed and spread on SD - Ura plates after overnight cultivation with uracil. From approximately $1 \times 10^{7}$ cells spread on the SD - Ura plate, 28 colonies were formed (Figure 6A). Ten of these colonies were randomly selected, and fluorescence intensity was measured. Values were compared to that of the parental MATa/ $\alpha$ cells (harboring $\mathrm{pH} 2 \mathrm{G}$ -
Pa1), which were used as a negative control (labeled as "Ctrl" in Figure 6B). All of the isolates derived from the SD - Ura plates exhibited higher fluorescence than Ctrl, suggesting that the proposed selection system for a-type of cells rarely produced false-positives. We selected the colony (No. 3) with the highest fluorescence intensity and performed another round of colony isolation to remove the cells of all plasmids. The resulting strain was designated BY4743A (Table 1).

Next, MATa $/ \alpha$ cells possessing both plasmids pHS-HoU and pL3G-2 $\alpha$ were washed and spread on the SD - Ura plates after overnight cultivation with uracil, as shown in Figure 1B. From approximately $1 \times 10^{6}$ cells spread on the SD - Ura plate, 23 colonies were formed (Figure 6C). Ten of these colonies were randomly selected, and fluorescence intensity was measured. Values were compared to that of the parental MATa/ $\alpha$ cells (harboring pL3G-2 $\alpha$ ), which were used as a negative control (labeled as "Ctrl" in Figure 6D). All of the colonies isolated on the SD - Ura plates exhibited higher fluorescence than Ctrl, suggesting that the proposed selection system for $\alpha$-type of cells rarely produced false-positives. We selected the colony (No. 4) with the highest fluorescence intensity and performed another round of colony isolation to remove the cells of all plasmids. The resulting strain was designated BY4743AL (Table 1).

To verify the mating ability of BY4743A and BY4743AL, mating assays $[20,21]$ were carried out as follows. Because BY4743A and BY4743AL have the same auxotrophy as BY4743, pH2G-Pa1 (containing HIS3 marker) was introduced into BY4743A (a-type) and BY4743 (MATa/ $\alpha$; parental strain), and pL3G-2 $\alpha$ (containing LEU2 marker) was introduced into BY4743AL ( $\alpha$-type) and BY4743. This allowed us to select zygotes using solid medium lacking histidine and leucine. The mating assays revealed successful mating between BY4743A and BY4743AL, but not between the two kinds of BY4743 transformants (Figure 6E). These results confirmed the validity of our proposed scheme. We believe that our approach will find application in manufacturing mating strains for yeast crossbreeding.

\section{Discussion}

The aim of this study was to establish a versatile method, using growth-based selection, for the isolation of mating strains for yeast crossbreeding (Figure 1). Although mating strains are traditionally prepared by sporulation from $M A T a / \alpha$ yeast strains used for industrial purposes, there are numerous strains that have extreme difficulty sporulating [7]. Alternative approaches using $\mathrm{HO}$ endonuclease have been developed in order to provide mating strains without sporulation $[18,22]$. Unfortunately, however, these approaches cannot be applied to yeast strains containing the "stuck" mutation (a single 
A

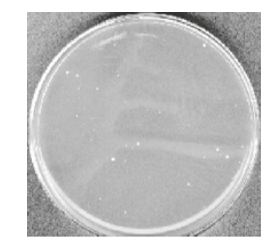

Screening of a-type of cells

C

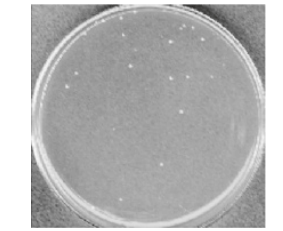

Screening of $\alpha$-type of cells
B

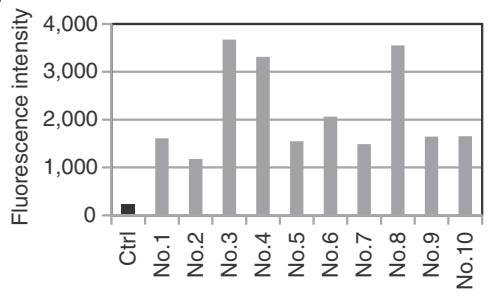

$\mathrm{D}$

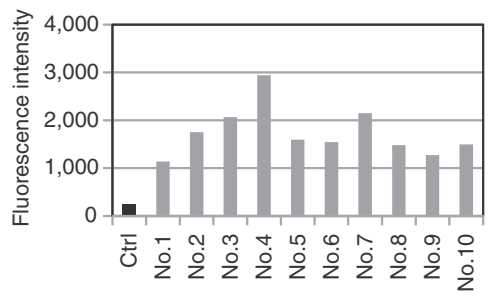

E $\quad$ BY4743 BY4743A

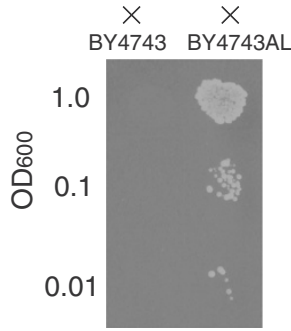

Figure 6 Isolation of a- or a-type of cells generated via spontaneous chromosomal aberration. (A) Direct images of colony formation in isolation of a-type of cells. Cell suspensions containing approximately $1 \times 10^{7}$ yeast cells ( $1 \mathrm{~mL}$ of suspension at $\left.\mathrm{OD}_{600} 1.0\right)$ were spread on selective solid medium (SD - Ura plate). (B) Fluorescent reporter assay to quantify expression of the GFP reporter gene in yeast transformants possessing plasmid pH2G-Pa1 ( $P_{\text {STE2 }}$ GFP). Ctrl indicates the parental MATa/a transformant (negative control). (C) Direct images of colony formation in isolation of a-type of cells. Cell suspensions containing approximately $1 \times 10^{6}$ yeast cells $\left(1 \mathrm{~mL}\right.$ of suspension at $\left.\mathrm{OD}_{600} 0.1\right)$ were spread on selective solid medium.

(D) Fluorescent reporter assay to quantify expression of the GFP reporter gene in yeast transformants possessing plasmid pL3G-2a (PSTE3-GFP). Ctrl indicates the parental MATa/a transformant (negative control). (E) Mating assays to investigate the mating abilities of yeast cells. Each spot corresponds to $10 \mu \mathrm{L}$ of suspension at the indicated $\mathrm{OD}_{600}$.

base substitution) at the MAT locus [23]. Here we focused on chromosomal aberration such as LOH and chromosome loss, alternative processes that produce aand $\alpha$-type of cells from parental MATa/ $\alpha$ cells. Although chromosomal aberration is remarkably tolerant of slight differences in the base sequences at the MAT locus, spontaneous frequencies of such events are less than $1 \times 10^{-4}$.

Hashimoto et al. used UV irradiation to increase the frequency of LOH [16], and Whittaker et al. used 12 kinds of chemicals to increase the frequency of chromosome loss [17]; however, these treatments are likely to randomly induce additional mutations and loss of other chromosomes. Because the purpose of yeast crossbreeding is to combine favorable traits of parental strains, these undesirable changes would be a concern for the resulting a- or $\alpha$-type of cells. To isolate a- and $\alpha$-type of cells generated via spontaneous chromosomal aberration in the absence of mutagens, we describe here the development of mating-type-dependent URA3 gene expression systems (Figure 1).

Generally, chromosomal stability becomes diminished by an increase in ploidy (e.g. less stable in triploid and tetraploid than in diploid cells). According to past report, the frequency of losing chromosome VII was approximately 10,000-fold higher in tetraploid than in diploid cells of S. cerevisiase [15]. In principle, mating-typedependent screening cannot exclude triploid, tetraploid and other higher polyploid cells that may emerge via chromosome aberration following autopolyploidization (mating between the a- and $\alpha$-type of cells generated from the same parental strain). Although these cells would presumably be available for yeast crossbreeding, chromosome stability of the hybrids should inevitably become diminished due to unneeded increase in ploidy. Hence, we utilized artificial formation of the a1- $\alpha 2$ complex [18] to remove the potential risk of autopolyploidization.

To develop growth selection systems for mating strains, we initially adopted $P_{\text {STE2 }}$ (for isolation of a-type of cells) and $P_{\text {STE3 }}$ (for isolation of $\alpha$-type of cells as the promoters to express the URA3 selection marker gene. By the use of YCp-type of vector, $P_{S T E 2}-U R A 3$ successfully permitted only a-type of yeast cells to grow without uracil. Compared to $P_{\text {STE2 }}$, regulation of gene expression under the control of $P_{\text {STE3 }}$ was leaky, a fact that had not been noted in a previous study using the GFP reporter gene [18]. Although we attempted to reduce leakiness of the URA3 gene expression by inclusion of the artificially formed a1- $\alpha 2$ complex (see Additional file 1: Figure S1C and D), little change was seen in the $\mathrm{OD}_{600}$ value of MATa and MATa/ $\alpha$ cells after 24. h cultivation (see Additional file 1: Figure S1D; compare to Figure 3D).

The difference in leakiness between $P_{\text {STE2 }}$ and $P_{\text {STE3 }}$ may reflect the distinct regulatory mechanisms controlling asg and $\alpha s g$ promoters. In $M A T \alpha$ and $M A T a / \alpha$ cells, expression of asg is suppressed by the $\alpha 2$ repressor 


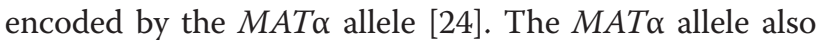
encodes the $\alpha 1$ activator, which facilitates the expression of $\alpha s g$. Whereas MATa cells rarely express $\alpha s g$ due to absence of the $\alpha 1$-coding gene within the MAT locus, $M A T a / \alpha$ cells are unlikely to express $\alpha s g$ because the $\alpha 1-$ coding gene is repressed by the a1- $\alpha 2$ complex (a1 is encoded by the MATa allele) [19]. Unlike promoters of asg, there is no component that can directly repress promoters of $\alpha s g$, which might permit a low level of basal (leaky) expression. In the present work, we succeeded in tight $\alpha$-type-specific URA3 gene expression by utilizing $P_{H O}$, a promoter of $h s g$ that is directly repressed by the a1- $\alpha 2$ complex (Figure 4).

While use of auxotrophic markers has an advantage in transformation efficiencies, the utility of them is limited within laboratory strains or genetically modified strains because industrial yeast strains usually do not have auxotrophic mutations such as ura3, his3 and leu2. However, in principle, our system can adopt drug-resistance markers which are available for industrial yeast strains. Instead of the URA3 marker, we attempted to use kanMX4 marker (see Additional file 2) that gives resistance for geneticin (G418), and succeeded in growing only target cells in cultivation medium containing G418 (see Additional file 3: Figure S2).

To evaluate the availability of the established growth selection systems, we manufactured a- and $\alpha$-type of cells as shown in Figure 1. The appearance frequencies of a- and $\alpha$-type of cells were $2.8 \times 10^{-6}$ and $2.3 \times 10^{-5}$, respectively. Because the frequency of chromosomal aberration generally varies by yeast strain, genetic modifications might have some effect on the frequency of chromosomal aberration in the parental BY4743 strain. Next, we verified the mating-type of colonies isolated on SD - Ura plates using GFP reporter gene expression. All colonies had the expected mating-type, suggesting the utility of isolating a- and $\alpha$-type of cells via the constructed growth-based selection systems.

We performed another round of colony isolation to remove all plasmids from yeast cells, yielding BY4743A and BY4743AL strains, respectively. To identify the event generating BY4743A or BY4743AL strain, we carried out quantitative analysis for the number of chromosome III (containing MAT genes) using real-time PCR (see Additional file 4: Figure S3). BY4743A strain has only 1 set of chromosome III, suggesting that it was generated via chromosome loss. On the other hand, BY4743AL strain has 2 sets of chromosome III, suggesting that it was generated via $\mathrm{LOH}$.

Furthermore, to confirm the stability of their mating ability, we carried out mating assays after serial passage in culture (see Additional file 5: Figure S4). Even after three passages, BY4743A and BY4743AL continued to exhibit equivalent levels of mating. We expect that the mating abilities of the generated a- and $\alpha$-type of cells will remain extremely stable, given that additional chromosomal rearrangements are unlikely to occur at the $M A T$ loci.

\section{Conclusion}

We have established a new approach to manufacture aand $\alpha$-type of cells from parental MATa/ $\alpha$ cells using growth selection systems. Use of spontaneous chromosomal aberration is quite beneficial in acquisition of mating strains inheriting desirable properties of industrially-used strains. We showed that yeast strains generated via spontaneous chromosomal aberration have stable mating ability, providing hybrid strains for use in yeast crossbreeding. Use of our method should help promote further advances of yeast-based biosynthesis approaches and in other experimental areas of research such as quantitative trait locus analysis and genome-wide association studies that permit the linking of phenotypic traits and genotypic data.

\section{Methods}

\section{Strains and media}

Detailed information about Saccharomyces cerevisiae strains BY4741, BY4742, and BY4743 [25], as well as other strains used in this study, is shown in Table 1. Yeast cells were grown in YPD medium ( $1 \%$ yeast extract, $2 \%$ peptone and $2 \%$ glucose) or in $\mathrm{SD}$ medium $(0.67 \%$ yeast nitrogen base without amino acids (Becton Dickinson and Company, Franklin Lakes, NJ, USA) and $2 \%$ glucose). A final concentration of $2 \%$ agar was added to make solid media.

\section{Construction of plasmids}

The sequences of oligonucleotides used in this study are shown in Table 2. The plasmids shown in Table 1 were made as follows. Using pRS316 (provided by the National BioResource Project (NBRP) of the MEXT, Japan) as a template, the URA3 gene was amplified with oligonucleotide pair $\mathrm{o} 1$ and $\mathrm{o} 2$, and inserted in place of the EGFP at the NotI-BamHI sites of pHY-2GA [18], yielding a YEptype plasmid designated pHY-2U. Similarly, using pRS316

Table 2 Sequences of oligonucleotides used to construct
plasmids
\begin{tabular}{ll}
\hline Number & Sequence \\
\hline 1 & $5^{\prime}$-gaatcaaaaGCGGCCGCatgtcgaaagctacatat-3' \\
2 & $5^{\prime}$-ccccagtttgGGATCCttagttttgctggccgcat-3' \\
3 & $5^{\prime}$-aaaattttcGCGGCCGCatgtcgaaagctacatat-3' \\
4 & $5^{\prime}$-aattggagctccaCCGCGG-3' \\
5 & $5^{\prime}$-cgggccccccCTCGAG-3' \\
6 & $5^{\prime}$-aattggagctccaCCGCGGcatttttgtttctttggac-3' \\
7 & $5^{\prime}$-tttcgacatGCGGCCGCtttaaagtatagatagaa-3' \\
\hline
\end{tabular}


as a template, the URA3 gene was amplified with oligonucleotide pair o3 and o2, and inserted in place of the $E G F P$ at the NotI-BamHI sites of pLY-3GC [18], yielding a YEp-type plasmid designated pLY-3U.

The YCp-type plasmids used to express the URA3 gene were constructed as follows. DNA fragments containing $P_{\text {STE2 }}$-URA3 were amplified from $\mathrm{pHY}-2 \mathrm{U}$ using oligonucleotide pair o4 and o5, and inserted into the SacII-XhoI sites of pRS315 (provided by NBRP), yielding the plasmid pLS-2U. DNA fragments containing $P_{S T E 3}-U R A 3$ were amplified from pLY-3U using oligonucleotide pair o4 and o5, and inserted into the SacII-XhoI sites of pRS313 (provided by NBRP), yielding the plasmid pHS-3U. The haploid-type-specific promoter, $P_{H O}$, was amplified using oligonucleotide pair 06 and 07 from genomic DNA derived from strain BY4741, and inserted in place of $P_{\text {STE3 }}$ at the SacII-NotI sites of pHS-3U, yielding the plasmid pHSHoU. Each plasmid was introduced into yeast cells using the lithium acetate method [26].

\section{Investigation of cell growth characteristics}

Each yeast transformant was grown in $500 \mu \mathrm{L}$ of SD medium without or with $20 \mathrm{mg} / \mathrm{L}$ uracil at $30^{\circ} \mathrm{C}$, setting initial optical density at $600 \mathrm{~nm}\left(\mathrm{OD}_{600}\right)$ at 0.03 . The $\mathrm{OD}_{600}$ values of cultures were monitored using a UV/visible spectrophotometer (Ultrospec 3100 pro; GE Healthcare Japan Corporation, Tokyo, Japan).

\section{Fluorescent reporter assay}

The EGFP gene was used as a fluorescent reporter to indicate mating type. Reporter-containing cells were incubated at $30^{\circ} \mathrm{C}$ for $18 \mathrm{~h}$, harvested and washed with distilled water. The cells then were resuspended in $100 \mu \mathrm{L}$ of distilled water to an $\mathrm{OD}_{600}$ of 5.0. GFP fluorescence intensities were measured using an Infinite 200 fluorescence microplate reader (Tecan Japan Co., Ltd., Kawasaki, Japan). For detection of the GFP signal, the excitation wavelength was set at $485 \mathrm{~nm}$ with a bandwidth of $20 \mathrm{~nm}$, and the emission wavelength was set at $535 \mathrm{~nm}$ with a bandwidth of $25 \mathrm{~nm}$. The gain was set at 50 .

\section{Mating assay}

Evaluation of mating ability was performed as follows. Yeast diploid cells were cultivated with the mating partner in $1 \mathrm{ml}$ of YPD medium at $30^{\circ} \mathrm{C}$ for $1.5 \mathrm{~h}$, with an initial $\mathrm{OD}_{600}$ of 0.1. After cultivation, yeast cells were harvested, washed, and resuspended in distilled water. Starting from an initial $\mathrm{OD}_{600}$ of $1,0.1$, or $0.01,10 \mu \mathrm{l}$ of cell suspensions were spotted on SD solid medium without the appropriate amino acids for growth selection of zygotes. After incubation at $30^{\circ} \mathrm{C}$ for 2 days, the image data was recorded for colonies on solid medium.

\section{Isolation of yeast cells with target mating-type}

Parental MATa $/ \alpha$ cells were grown in $500 \mu \mathrm{L}$ of SD media with $20 \mathrm{mg} / \mathrm{L}$ uracil at $30^{\circ} \mathrm{C}$ for $18 \mathrm{~h}$, and then harvested and washed with distilled water. The cells then were resuspended in distilled water. Cell suspensions were spread on SD - Ura plates.

\section{Additional files}

\begin{abstract}
Additional file 1: Figure S1. Alternative growth selection systems for isolation of a-type or a-type yeast cells by formation of the a1-a2 complex. (A) Plasmids used for a-type-specific URA3 gene expression. The plasmid pLS-2U was used in combination with $\mathrm{pH} 2 \mathrm{G}-\mathrm{Pa} 1$, which suppresses the mating ability of a-type cells. (B) The $\mathrm{OD}_{600}$ values of cultures of double transformants (harboring both plasmids pLS- $2 \mathrm{U}$ and pH2G-Pa1) at 24 h cultivation. Black bars indicate cultivation with uracil, and gray bars indicate cultivation without uracil. (C) Plasmids used for a-type-specific URA3 gene expression. The plasmid pHS-3U was used in combination with pL3G-2a, which is required for suppressing the mating ability of a-type cells. (D) The $\mathrm{OD}_{600}$ values of cultures of double transformants (harboring both plasmids pHS-3U and pL3G-2a) at $24 \mathrm{~h}$ cultivation. Black bars indicate cultivation with uracil, and gray bars indicate cultivation without uracil.
\end{abstract}

Additional file 2: Supporting information for Materials and Methods. Table S1. Sequences of oligonucleotides used to construct plasmids.

Additional file 3: Figure S2. Growth of yeast transformants harboring kanMX4 selection marker gene. (A) Plasmid map of pLS-2 K containing CEN6/ARSH4 origin of replication (providing cellular retention of single-copy plasmids) and $P_{\text {STE2 }}-$ KanM $X 4$ construct (activated in a-type yeast cells). (B) The growth curves of pLS-2 K transformants. Closed symbols indicate cultivation without $\mathrm{G} 418$, and open symbols indicate cultivation with G418. (C) Plasmid map of pHS-HoK-2a containing CEN6/ARSH4 origin of replication and $P_{H O}-k a n M X 4$ construct combined with $P_{\text {STE2 }}-a 2$ construct (activated in a-type yeast cells). (D) The growth curves of pHS-HoK-2a transformants. Symbols are as in B.

Additional file 4: Figure S3. Ploidy analysis using real-time PCR. The normalized copy number of the PGK1 gene is an indicator of ploidy (A) for BY4743A and (B) for BY4743AL strains. Standard deviations of three replicates are presented.

Additional file 5: Figure S4. Investigation of stability of the mating abilities of yeast cells after serial passage of cultures. Up to three serial passages were carried out, and then the resulting BY4743, BY4743A, and BY4743AL transformants were used for mating assays.

\section{Abbreviations}

EGFP: Enhanced green fluorescent protein; LOH: Loss of heterozygosity; MAT locus: Mating-type locus; hsg: Haploid-specific genes; asg: a-type-specific genes; asg: a-type-specific genes.

\section{Competing interests}

We declare that all authors are inventors on a pending patent using aspects of this system.

\section{Authors' contributions}

NF designed the study, conducted experiments, analyzed data, and co-wrote the manuscript. SH analyzed data and co-wrote the manuscript. Both authors read and approved the final manuscript.

\section{Acknowledgements}

The plasmids pRS313, pRS315, and pRS316, were provided by the National BioResource Project (NBRP) of the MEXT, Japan. This work was supported by JSPS KAKENHI Grant Number 25820406.

Received: 8 July 2013 Accepted: 12 November 2013 Published: 21 November 2013 


\section{References}

1. Higgins VJ, Bell PJL, Dawes IW, Attfield PV: Generation of a novel Saccharomyces cerevisiae strain that exhibits strong maltose utilization and hyperosmotic resistance using nonrecombinant techniques. Appl Environ Microbiol 2001, 67:4346-4348.

2. Kishimoto M: Fermentation characteristics of hybrids between the cryophilic wine yeast Saccharomyces bayanus and the mesophilic wine yeast Saccharomyces cerevisiae. J Ferment Bioeng 1994, 77:432-435.

3. Shinohara T, Mamiya S, Yanagida F: Introduction of flocculation property into wine yeasts (Saccharomyces cerevisiae) by hybridization. J Ferment Bioeng 1997, 83:96-101.

4. Benitez T, Gasent-Ramirez JM, Castrejon F, Codon AC: Development of new strains for the food industry. Biotechnol Prog 1996, 12:149-163.

5. Guijo S, Mauricio JC, Salmon JM, Ortega JM: Determination of the relative ploidy in different Saccharomyces cerevisiae strains used for fermentation and 'flor' film ageing of dry sherry-type wines. Yeast 1997, 13:101-117.

6. Maráz A: From yeast genetics to biotechnology. Acta Microbiol Immunol Hung 2002, 49:483-491.

7. Gunge N: Breeding of bakers' yeast-determination of the ploidy and an attempt to improve practical properties. Japan J Genet 1966, 41:203-214.

8. Tsuboi M, Takahashi T: Genetic analysis of the nonsporulating phenotype of brewer's yeasts. J Ferment Technol 1988, 66:605-613.

9. Kumaran R, Yang SY, Leu JY: Characterization of chromosome stability in diploid, polyploid and hybrid yeast cells. PLoS One 2013, 8:e68094.

10. Andersen MP, Nelson ZW, Hetrick ED, Gottschling DE: A genetic screen for increased loss of heterozygosity. Saccharomyces cerevisiae 2008, 179:1179-1195.

11. Takagi Y, Akada R, Kumagai H, Yamamoto K, Tamaki H: Loss of heterozygosity is induced in Candida albicans by ultraviolet irradiation. Appl Microbiol Biotechnol 2008, 77:1073-1082.

12. Alvaro D, Sunjevaric I, Reid RJ, Lisby M, Stillman DJ, Rothstein R: Systematic hybrid LOH: a new method to reduce false positives and negatives during screening of yeast gene deletion libraries. Yeast 2006, 23:1097-1106.

13. Daigaku Y, Endo K, Watanabe E, Ono T, Yamamoto K: Loss of heterozygosity and DNA damage repair in Saccharomyces cerevisiae. Mutat Res 2004, 556:183-191.

14. Hiraoka M, Watanabe K, Umezu K, Maki H: Spontaneous loss of heterozygosity in diploid Saccharomyces cerevisiae cells. Genetics 2000, 156:1531-1548.

15. Mayer W, Aguilera A: High levels of chromosome instability in polyploids of Saccharomyces cerevisiae. Mutat Res 1990, 231:177-186.

16. Hashimoto S, Aritomi K, Minohara T, Nishizawa Y, Hoshida H, Kashiwagi S, Akada R: Direct mating between diploid sake strains of Saccharomyces cerevisiae. Appl Microbiol Biotechnol 2006, 69:689-696.

17. Whittaker SG, Zimmermann FK, Dicus B, Piegorsch WW, Resnick MA, Fogel S: Detection of induced mitotic chromosome loss in Saccharomyces cerevisiae-an interlaboratory assessment of 12 chemicals. Mutat Res 1990, 241:225-242.

18. Fukuda N, Matsukura S, Honda S: Artificial conversion of the mating-type of Saccharomyces cerevisiae without autopolyploidization. ACS Synth Biol 2013. in press.

19. Gelfand B, Mead J, Bruning A, Apostolopoulos N, Tadigotla V, Nagaraj V, Sengupta AM, Vershon AK: Regulated antisense transcription controls expression of cell-type-specific genes in yeast. Mol Cell Biol 2011, 31:1701-1709.

20. Fukuda N, Ishii J, Tanaka T, Kondo A: The competitor-introduced Gy recruitment system, a new approach for screening affinity-enhanced proteins. FEBS J 2010, 277:1704-1712.

21. Fukuda N, Ishii J, Kondo A: Gy recruitment system incorporating a novel signal amplification circuit to screen transient protein-protein interactions. FEBS J 2011, 278:3086-3094.

22. Kanai K, Kobayashi O: Method for imparting mating ability to yeast. Japanese Kokai Tokkyo: Koho patent publication number JP 2010-220481. Japanese Kokai Tokkyo Koho Application number JP 2009-068013, Date 2009.03.19.

23. Ray BL, White Cl, Haber JE: Heteroduplex formation and mismatch repair of the "stuck" mutation during matingtype switching in Saccharomyces cerevisiae. Mol Cell Biol 1991, 11:5372-5380.
24. Botstein D: Ira Herskowitz: 1946-2003. Genetics 2004, 166:653-660.

25. Brachmann CB, Davies A, Cost GJ, Caputo E, Li J, Hieter P, Boeke JD: Designer deletion strains derived from Saccharomyces cerevisiae S288C: a useful set of strains and plasmids for PCR-mediated gene disruption and other applications. Yeast 1998, 14:115-132.

26. Gietz D, St Jean A, Woods RA, Schiestl RH: Improved method for high efficiency transformation of intact yeast cells. Nucleic Acids Res 1992, 20:1425.

doi:10.1186/1754-1611-7-27

Cite this article as: Fukuda and Honda: Development of growth selection systems to isolate a-type or a-type of yeast cells spontaneously emerging from MATa/a diploids. Journal of Biological Engineering 2013 7:27.

\section{Submit your next manuscript to BioMed Central and take full advantage of:}

- Convenient online submission

- Thorough peer review

- No space constraints or color figure charges

- Immediate publication on acceptance

- Inclusion in PubMed, CAS, Scopus and Google Scholar

- Research which is freely available for redistribution 